\title{
CHANGES IN THE MORPHOLOGY OF INTERSTELLAR ICE ANALOGUES AFTER HYDROGEN ATOM EXPOSURE
}

\author{
E. Congiu ${ }^{1}$, M. Accolla ${ }^{1,2}$, F. Dulieu ${ }^{1}$, H. Chaabouni ${ }^{1}$, J.-L. Lemaire $^{1}$, \\ H. Mokrane ${ }^{1}$, E. Matar ${ }^{1}$, G. Manicò ${ }^{2}$ and V. Pirronello ${ }^{2}$
}

\begin{abstract}
Interstellar water ice is mainly amorphous, but the nature of its morphology still remains poorly known. The experimental study described in this work focuses on how relevant changes of the ice morphology result from atomic hydrogen exposure and subsequent recombination. We show that there is an exponential decrease in the porosity of the amorphous water ice sample following hydrogen-atom irradiation. These and other laboratory results lead us to suggest that water ice in space is almost certainly amorphous and non-porous (compact).
\end{abstract}

\section{Introduction}

Amorphous solid water (ASW) is reputed to be the most abundant form of water in the Universe, thanks to its propensity for forming, molecule after molecule, as a deposit on interstellar dust particles (Jenniskens et al. 1995; Austen Angell 2004). The accretion of icy mantles (predominantly constituted by ASW) on silicate and carbonaceous dust grains takes place in interstellar molecular clouds. Some of them are cold $(\sim 10 \mathrm{~K})$ and dense $\left(10^{4}-10^{6} \mathrm{~cm}^{-3}\right)$ regions where gaseous species heavier than hydrogen and helium freeze out onto the grains. The most important and abundant molecular species, $\mathrm{H}_{2}$, is formed thanks to reactions occurring on amorphous water ice in molecular clouds (Hollenbach \& Salpeter 1971; Manicò et al. 2001). As for $\mathrm{H}$ atoms, it has been evaluated that, in dense clouds, the average number density ratio of the atomic hydrogen component compared to the molecular one is $\sim 0.1 \%$ (Li \& Goldsmith 2003). In a dark cloud, atomic hydrogen thus represents the third most abundant gas-phase species, after $\mathrm{H}_{2}$ and He. Along with atoms and molecules available in the gas-phase and the condensed species on

\footnotetext{
1 LERMA-LAMAp, UMR 8112 du CNRS, Observatoire de Paris et Université de Cergy-Pontoise, 5 mail Gay-Lussac, 95000 Cergy Pontoise Cedex, France; e-mail: econgiu@u-cergy.fr

2 DMFCI, Università di Catania, Viale Doria 6, 95125 Catania, Italy
} 
the surface of dust grains, the roughness of the icy grains can also greatly affect the efficiency of surface reactions. In fact, the morphology of amorphous water ice in the ISM appears to be the crucial parameter to thoroughly understand the energetics and dynamics of gas-grain reactions in molecular clouds. Laboratory simulations have indeed confirmed that interstellar porous ice analogues can be compacted quite efficiently by UV irradiation (Palumbo et al. 2010) and by cosmic ion bombardment (Palumbo 2006; Raut et al. 2008) in a time comparable to the estimated ice mantle lifetime. Similarly, amorphous water ice exhibits a compact structure when it is formed by simulating water formation in space at low temperatures via surface reactions between $\mathrm{O}_{2}$ and $\mathrm{H}$-atoms (Oba et al. 2009). The present study is intended to provide another piece of evidence that porous ASW undergoes a compaction process also as a consequence of surface $\mathrm{H}+\mathrm{H}$ reactions leading to molecular hydrogen formation.

\section{Results}

The compaction of porous ASW ice due to deuterium atom irradiation is investigated for various thickness of the ice film (Accolla et al. 2011). TPD spectra of a fraction of a monolayer $\left(1 \mathrm{ML}=10^{15}\right.$ molecules $\left.\mathrm{cm}^{-2}\right)$ of $\mathrm{D}_{2}$ molecules were obtained before and after D-atom exposure of the porous ASW film to probe its morphology and the possible loss of porosity as a function of D-atom fluence. Figure 1a shows the effects of D-atom exposure on the morphology of a 4-ML porous ASW film. The solid line represents a $\mathrm{D}_{2}$ desorption spectrum from compact ice, while open squares show the $\mathrm{D}_{2}$ desorption trace from a non-irradiated 4-ML porous ASW ice. The other TPD spectra of Figure 1a show $\mathrm{D}_{2}$ desorption after subsequent D-atom bombardment of the porous ice sample kept at $10 \mathrm{~K}$. Increasing the D-atom fluence, it is apparent a progressive shift of the desorption peaks towards lower temperatures, as indicated by the arrows.

Figure 1b summarizes all the experiments performed in the present study, i.e., for porous ice thickness $m$ of $1 \mathrm{ML}, 2 \mathrm{ML}, 4 \mathrm{ML}, 6.5 \mathrm{ML}$ and $8 \mathrm{ML}$. In Figure 1b, we show the normalized fraction of $\mathrm{D}_{2}$ molecules with adsorption energy greater than $66 \mathrm{meV}$ (corresponding adsorption energy of molecules coming off at a surface temperature greater than $22.5 \mathrm{~K}$ ). We quantified the porosity of each ASW ice sample as the surface area subtended by the TPD trace for temperatures greater than $22.5 \mathrm{~K}$, since in this range the desorbing molecules were almost certainly adsorbed on "porous" binding sites. For each ice thickness, the number of molecules that can adsorb on the surface decreases gradually as the D-atoms fluence is increased on the porous ice sample. It is clear that the experimental curves of each ice thickness are well fitted by a one-parameter (the D-atom fluence $\Phi_{c, m}$ ) exponential decrease. We find a linear correlation between the ice thickness and the characteristic fluence $\Phi_{c, m}$. From the slope of the linear fit, we calculated a characteristic fluence $\Phi_{0}=2.2 \times 10^{16} \mathrm{D}$-atoms $\mathrm{cm}^{-2}$ per $\mathrm{H}_{2} \mathrm{O} \mathrm{ML}$, namely, the hydrogen atom fluence needed to destroy the ice porosity of one layer of highly porous water ice. 

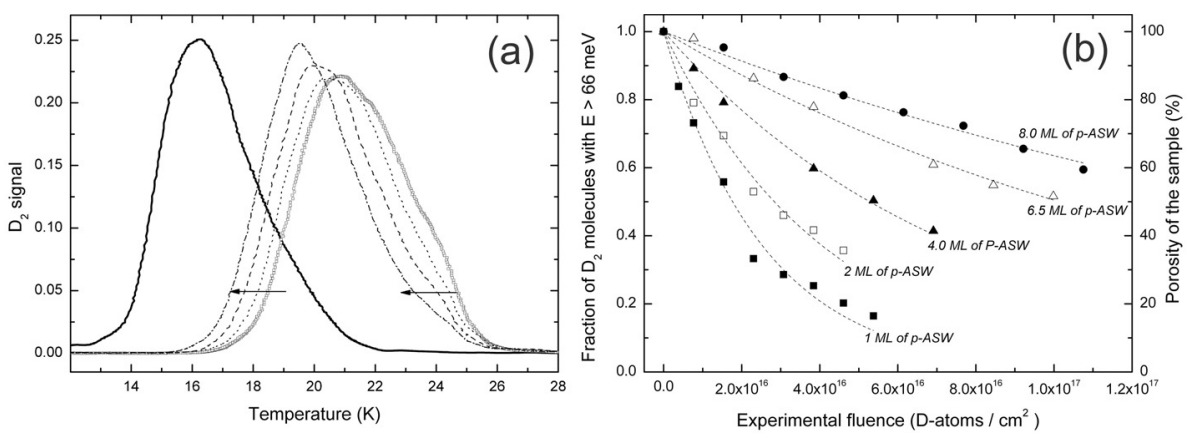

Fig. 1. (a) Normalized TPD spectra of $0.11 \mathrm{ML}$ of $\mathrm{D}_{2}$ from compact ASW (solid line) and of 0.17 ML of $\mathrm{D}_{2}$ from a 4-ML porous ASW film as deposited (open squares), after 64 min (dotted line), after 224 min (dashed line) and $288 \mathrm{~min}$ (dash-dot line) of D-atom irradiation. (b) Normalized fraction of $\mathrm{D}_{2}$ molecules that can adsorb on the porous ice surface with an energy greater than $66 \mathrm{meV}$ vs. fluence of D-atoms. Data-points of the 4-ML curve, for example, can be directly compared with the curves in Figure 1a to make a quantitative estimate of the loss of porosity with time.

\section{Astrophysical implications}

The icy mantles covering dust grains in dark clouds are believed to be $40-100$ molecular layers thick. The interstellar flux of hydrogen atoms impinging on dust grains can be calculated as follows:

$$
\phi_{H}=\frac{1}{4} n_{H} v_{H}
$$

where $n_{H}$ is the H-atom density in gas phase and $v_{H}=\sqrt{8 k T / \pi m}$ is the mean velocity of an hydrogen atom. Given a typical molecular cloud with $n_{H}=2.3 \mathrm{~cm}^{-3}$

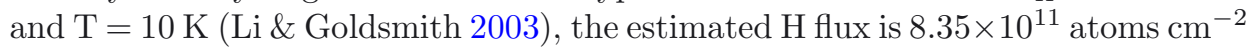
$\mathrm{yr}^{-1}$. In Figure 2 we present the evolution of a porous film of water ice in a typical molecular cloud. The dashed line represents the icy mantle thickness following a mean formation rate of $100 \mathrm{ML}$ in $10^{6}$ years (Cuppen \& Herbst 2007). The solid line shows the evolution of the thickness of the porous layer as it is concurrently exposed to the mean $\mathrm{H}$-atom flux as calculated above and undergoes a porosity destruction rate calculated using the characteristic fluence $\Phi_{0}$ found in this work. Figure 2 shows that, due to $\mathrm{H}$-atom irradiation, the porous film never exceeds a thickness of $55 \mathrm{ML}$ and it is completely destroyed within $3 \times 10^{6}$ years. Nevertheless, this length of time ought to be considered as an upper limit and a rather conservative estimate since the mean formation rate of water ice employed is based on two very optimistic hypotheses: 1) a very high efficiency of conversion to water by surface reactions and 2) water ice just formed is assumed to be porous. Moreover, should the H-atom flux be a factor of 2 higher or the actual formation rate be a factor of two slower, the thickness of the porous layer would never exceed 


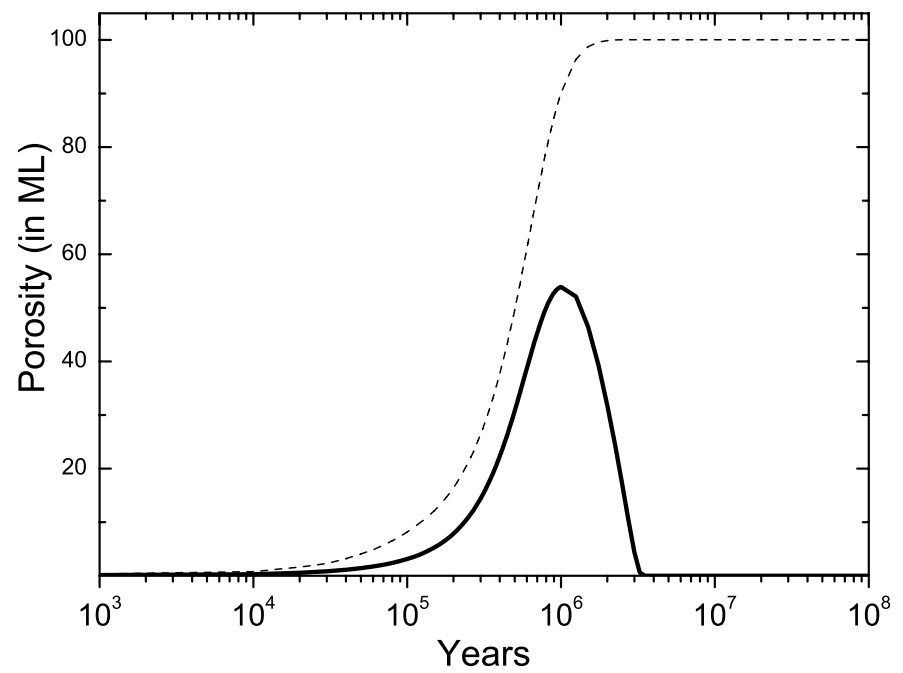

Fig. 2. Evolution of the thickness of a highly porous ASW mantle following grain surface reactions only (dashed line) and with concurrent $\mathrm{H}$-atom exposure (solid line) in a typical molecular cloud.

$10 \mathrm{ML}$ and would be completely compacted in less than $10^{6}$ years. Therefore, the destruction of porosity is completed in a lapse of time much shorter than the estimated lifetime of a molecular cloud $\left(3 \times 10^{7}-5 \times 10^{8}\right.$ years $)$.

\section{References}

Accolla, M., et al., 2011, Phys. Chem., Chem. Phys., 13, 8037

Austen Angell, C., 2004, Annu. Rev. Phys. Chem., 55, 559

Cuppen, H.M., \& Herbst, E., 2007, ApJ, 668, 294

Hollenbach, D., \& Salpeter, E.E., 1971, ApJ, 163, 155

Jenniskens, P., et al., 1995, ApJ, 455, 389

Li, D., \& Goldsmith, P.F., 2003, ApJ, 585, 823

Manicò, G., et al., 2001, ApJ, 163, 155

Oba, Y., et al., 2009, ApJ, 701, 464

Palumbo, M.E., et al., 2010, J. Mol. Struct., 972, 64

Palumbo, M.E., 2006, A\&A, 453, 903

Raut, U., et al., 2008, ApJ, 687, 1070 